\title{
Le frontalier. Regard sociologique sur un être méconnu
}

Hans Leo Krämer

\section{OpenEdition}

\section{Journals}

Édition électronique

URL : http://journals.openedition.org/ress/403

DOI : $10.4000 /$ ress.403

ISSN : 1663-4446

Éditeur

Librairie Droz

Édition imprimée

Date de publication : 1 mars 2004

Pagination : 199-218

ISBN : 2-600-00941-8

ISSN : 0048-8046

\section{Référence électronique}

Hans Leo Krämer, «Le frontalier. Regard sociologique sur un être méconnu », Revue européenne des sciences sociales [En ligne], XLII-129 | 2004, mis en ligne le 05 novembre 2009, consulté le 02 mai 2019. URL : http://journals.openedition.org/ress/403 ; DOI : 10.4000/ress.403 


\section{Hans Leo KRÄMER}

\section{LE FRONTALIER. REGARD SOCIOLOGIQUE SUR UN ÊTE MÉCONNU}

Le livre de Philippe Besnard Mours et humeurs des Français au fil des saisons (1989), que j'ai lu à l'époque comme un roman fascinant, dépeint un tableau des activités et des comportements des Français dans un langage et un style clairs et vivants. Bien que la méthode utilisée soit classique, exploitant des données statistiques et empiriques sur le Français en tant qu' «animal saisonnier» typique, il m'est venu l'idée qu'il serait intéressant de comparer ce Français-là à un autre type de Français. Est-ce que les «effets de la ronde annuelle des saisons » subiraient une modification si on les étudiait auprès de Français qui sont régulièrement en contact avec une autre culture? Mon hypothèse est qu'il existe une variation selon que la ronde annuelle des saisons est croisée au moins partiellement par d'autres rythmes et rites saisonniers comme on les pratique en Allemagne. Les frontaliers lorrains qui circulent tous les jours entre deux pays et deux cultures différents, pourraient constituer les sujets idéaux d'une telle recherche.

On peut élargir le thème et faire la comparaison des modes de vie des Allemands de la Sarre avec ceux des travailleurs frontaliers et des Lorrains «sédentaires». Quand on sait qu'entre les deux régions «il y a un grand potentiel de rapport, de mélanges et de pénétration de cultures voisines » (Brücher, Dörrenbächer, 2000, p. 17), on peut supposer une certaine mixité franco-lorraine-sarroise dans les modes de vie de nos frontaliers. En partant de cette idée de mixité, on devrait peut-être corriger certaines affirmations dont Henri Mendras nous donne un exemple amusant quand il écrit: «Chacun sait que les Allemands mangent plus de pain et de charcuterie que les Français et qu'ils préfèrent la bière au vin ». Et il ajoute très justement que manquent des études comparatives analytiques qui permettraient «d'esquisser une géographie et une véritable sociologie des modes de vie» (Mendras, 1997, p. 383).

Mon intérêt pour les frontaliers a aussi été stimulé par l'expérience de ma propre vie dont la plus grande partie s'est passée près de la frontière entre l'Allemagne et la France, c'est-à-dire entre la Sarre et la Lorraine. Marié à une française du Centre de la France, exilée au pays de l'ennemi de naguère, et vivant avec des enfants binationaux et bilingues, je sentais que des frontières extérieures et intérieures marquaient ma personnalité. Une fois devenu sociologue, j'ai commencé à observer les modes de vie des deux côtés de la frontière. S'il est relativement facile de conclure aux goûts culinaires différents en regardant les paniers des clients dans les supermarchés le long de la frontière, il est bien plus compliqué de comprendre les visions du monde des frontaliers. Le sociologue ne doit tout de même pas se contenter de généralités montrant que la vie matérielle de la France et de l'Allemagne «tourne à la symbiose», mais que, culturellement elles sont 
lointaines et que «spirituellement elles ne s'entendent pas»(Glucksmann, 1997). Philippe Besnard n'avait pas tout à fait tort en me présentant à ses collègues comme «un bon connaisseur» de la sociologie française, une louange bien trop flatteuse évidemment, mais justifiée peut-être par mon propre mode de vie (vivre la frontière) et mon inclination professionnelle (observer la frontière). On définit mon type comme «frontalier socioculturel» qui «joue l'intermédiaire et le traducteur entre différentes cultures» (Pankoke, 1999, p. 19).

Le sujet de mon intérêt est le groupe des frontaliers lorrains qui vont travailler quotidiennement dans la Sarre (Allemagne). Les statistiques de l'emploi y enregistrent une augmentation incessante du nombre de frontaliers depuis vingt ans. Un peu plus de 25000 personnes y travaillent dans des conditions régulières (contrat de travail, sécurité sociale). Il faut y ajouter au moins 5000 personnes travaillant en général sans protection sociale (p. ex. des femmes de ménage). Presque la moitié des frontaliers sont des femmes. À part ces quelques traits statistiques, le profil des frontaliers est assez flou, faute d'études sociologiques approfondies. D'après certaines sources (Gries et alii, 1997; CDR/ISOPLAN, 1996; EURES, 2000) la plus grande partie est embauchée dans des secteurs assez sensibles à la conjoncture (industrie automobiles, aciérie, industrie céramique, bâtiment). Un quart seulement travaille dans le secteur des services. Un fait (tristement) remarquable est que souvent de nombreux frontaliers sont engagés et rémunérés en-dessous de leurs qualifications et de leur formation scolaire et professionnelle.

Nous n'avons pratiquement pas d'informations sur tout ce qui concerne la socialisation, la construction de l'identité, les rapports entre les frontaliers et l'entourage allemand, etc. Que signifie pour eux «être frontalier»? Sentent-ils une influence de ce mode de vie frontalier sur leur vie privée? Se voient-ils comme des étrangers ou des «amis voisins» en Allemagne? Que pensent-ils de l'accueil sur leur lieu de travail et ailleurs? Où se situent-ils dans l'éventail politique? Comment voient-ils leur avenir? Beaucoup de questions et peu de réponses. Pour le moment le frontalier est une terra incognita. Je ne prétends pas pouvoir changer cette situation par mes réflexions marginales. Je veux tout simplement montrer une piste possible pour analyser ce type de frontalier.

\section{LA FRONTIÈRE EXISTE}

Le grand écrivain autrichien Joseph Roth notait dans une lettre d'octobre 1927 évoquant un voyage en train de Paris à Sarrebruck en Allemagne: «Je hais la «frontière » entre deux pays. C'est une notion trop vaste pour les réalités qu'elle désigne. Qu'est-ce qu'une «frontière»? Un poteau, un treillis en fil de fer, un douanier, un visa, un tampon, un arrêt. Au lieu d'être des symboles ce sont des infamies» (Roth, s.d., p. 11). Les Européens «intra muros» de Schengen ne connaissent plus ou presque ce rituel; seulement si un État, pour des raisons de sécurité, met en vigueur des plans de vigilance à ses frontières extérieures. Traverser des frontières intérieures dans l'Europe de la Communauté n'est plus un problème pour ceux qui sont «en règle». Un fait banal mais important pour les frontaliers, qui facilite le va-et-vient légal. On se rappelle que le Traité de Maastricht du 7 février 1992 voulait que l'Union Européenne crée «un espace sans 
frontières intérieures », réalisant ainsi «la liberté de mobilité ». Les passages clandestins des frontières n'existeront plus que dans la mémoire collective des Européens «normaux».

Néanmoins la frontière traditionnelle devenue aujourd'hui quasiment invisible reste existante et vivante. Rarement le discours frontalier, l'évocation « de la frontière » qu'il faudrait abolir aussi dans les consciences et dans les cœurs, se souvenir de son rôle important pour la construction de l'identité nationale et régionale, etc., n'a été si répandu et publiquement discuté qu'aujourd'hui. Michel Tournier (1998) a peut-être raison quand il lie ce phénomène à une renaissance des particularismes des «Provinces». G. Simmel l'a reconnu dans sa célèbre définition de la frontière en disant qu'elle est bien plus qu'un fait extérieur. «La frontière n'est pas un fait d'espace (räumliche Tatsache) avec des effets sociologiques, mais un fait sociologique qui se forme dans l'espace» (Simmel, 1908, p. 623). De récentes études sur la perception de la frontière entre les deux Allemagne, juridiquement abolie depuis juillet 1990, ont montré que des gens qui à l'époque vivaient près de la frontière, continuent à être conscients de l'ancienne «séparation ».

Même sentimentalement la frontière est ressentie comme une réalité spécifique. Interrogés à ce propos, les frontaliers lorrains affirment qu'ils éprouvent souvent une «drôle de sensation» quand ils traversent la frontière même invisible. Joseph Roth parle d'un «changement d'atmosphère» et il ajoute que même le rythme du wagon est autre et que «la locomotive tire selon d'autres mélodies». De nos jours, un jeune écrivain ukrainien, Juri Andruchowytsch, grand voyageur entre l'Est et l'Ouest, écrit un peu ironiquement que chaque fois qu'il traverse la frontière pour rentrer chez lui, le climat quasiment identique des deux côtés, «change subitement, il se détériore régulièrement, l'horizon s'obscurcit et des tonnes de pluie ou de neige tombent du ciel» (Andruchowytsch, 2003, p. 159).

Il est évident que la frontière politique et étatique est constitutive d'une conception sociologique du frontalier, car d'après le décret 140/71 de l'Union Européenne le frontalier est «chaque travailleur ou indépendant qui exerce sa profession sur le territoire d'un État Membre et qui habite sur le territoire d'un autre État Membre où il rentre normalement chaque jour - au moins chaque semaine».

\section{L'AUTRE MONDE}

Le frontalier n'est pas un simple travailleur qui vit «de l'autre côté», mais un représentant d'un «autre monde». Lucien Lefebvre emploie cette image dans sa définition de la frontière. Elle est frontière «si au-delà d'une ligne commence un autre monde, un rapport d'idées, de sentiments et de passions qui surprennent et déconcertent l'étranger» (Lefebvre, 1994, p. 163). Le frontalier est donc la concrétisation d'une autre culture, d'une autre langue. Mais sa langue peut aussi le trahir. Si le frontalier lorrain parle les deux langues, l'allemand d'ailleurs souvent dans sa version dialectique, dans la Sarre parfois on le prend pour «le petit cousin» lointain. Très souvent j'ai observé que ce petit cousin, maîtrisant fort bien l'allemand, changeait de tactique et ne parlait que le français que l'entourage allemand ne comprenait pas. Une telle ruse rétablit la «distinction» et sert de mécanisme pour garder et démontrer sa véritable identité. 
Il y a un grand problème de reconnaissance pour le frontalier lorrain. Son monde politique, social, culturel, etc. ressemble beaucoup à celui des Sarrois/Allemands. Cette parenté est à l'origine de malentendus et presque d'un paradoxe, car d'habitude le frontalier ne contredit pas si on le traite comme un «semblable». Mais, à la fois, il se sent quelque peu dévalorisé parce que son «altérité » n'est reconnue que partiellement. C'est probablement la raison pour laquelle le frontalier lorrain n'est pas non plus traité ouvertement comme un étranger véritable, par exemple comme un Turc. Celui-là au lieu de supports trouve un «champ d'aventures». L'expression est d'Alfred Schütz (1972). Les supports se basent sur un fond de rapports quotidiens d'une culture commune.

Tout de même «être frontalier» reste une aventure. D'abord parce qu'il n'est pas si simple de représenter un «autre monde» plein de préjugés envers les Allemands. Mais l'aventure réside principalement dans l'instabilité du statut général. En tant que Français il a été socialisé dans et pour la société française. Le succès de son activité à l'étranger ne dépend pas forcément de ses compétences mais beaucoup de forces qui lui sont extérieures, de la stabilité ou non-stabilité politique et économique de la Sarre. Structurellement on peut dire que l'intégration sociale du frontalier se passe hors de sa société d'origine, et en plus, cette intégration risque d'être résiliée. En outre l'intégration est incomplète parce qu'elle ne signifie pas automatiquement une intégration au système politique du pays de travail. «Citoyen» il ne peut l'être qu'en France. Le frontalier vit donc toujours entre inclusion et exclusion. Comment peut-il supporter la tension permanente qui en résulte? Je pense qu'il est obligé de développer une forte attitude de confiance, confiance dans la stabilité de l'ordre social et des institutions politico-économiques. P. Sztompa définit justement la confiance comme «une ressource culturelle indispensable pour réaliser le potentiel de l'action en pratique» (Sztompa, 1995, p. 255).

L'expression métaphorique de l'autre monde, de l'au-delà des frontières, se retrouve un peu partout dans la littérature de différentes disciplines. Des cinq modèles que j'ai construit deux seulement ont un rapport aux travailleurs frontaliers (Krämer, 2000). Le modèle du «marcheur et migrant» rassemble toutes les formes de passeurs de frontières. Le monde de son but (pays, société, culture, etc.) lui est relativement inconnu, c'est de l'autre monde qu'il attend la réalisation de ses rêves et de ses désirs, la satisfaction de ses besoins. Il veut y rester seulement pour un temps limité. Le modèle du «garde-voie» parle des frontaliers qui circulent le long d'une frontière comme des gardes inspectant leur terrain pour voir s'il y a des changements. Les lignes de frontières les attirent par une force invisible. Les deux modèles analysent les activités et les comportements des frontaliers dans la dimension espace. Ce n'est pas le cas par exemple du modèle des «deuxmondes »: ici les frontaliers sont des intellectuels. Ils circulent entre différentes positions idéologiques, entre le monde de l'art et de la science, entre «l'esprit» et «la matière». On rencontre ici aussi toutes sortes de «traîtres». Les curieux, ceux qui veulent connaître un autre monde, sont réunis dans mon modèle du «voyeur». Le frontalier s'approche d'une frontière pour jeter un coup d'œil de l'autre côté. L'écrivain sarrois Alfred Gulden l'exprimait ainsi: «Lorsqu'enfant, debout sur un bunker du Führer au bout du jardin de mes parents, je regardais au-dessus de Sarrelouis sur la ligne des collines, je me suis toujours demandé: qu'y a-t-il derrière?». 


\section{LE CADRE THÉORIQUE}

La sociologie est la science des frontières et des limites par excellence. Elle part de la prémisse que l'action humaine et sociale est limitée par de multiples forces qui agissent souvent à l'état latent et par des conditions sociales contraignantes. Peter Wagner a très bien reconstruit l'histoire de la délimitation des frontières comme un processus nécessaire pour construire une identité propre. Les différentes sociologies, la sociologie générale et les sociologies spéciales qui s'intéressent rarement au cas concret du frontalier concret, peuvent tout de même donner un cadre analytique à notre sujet. J'ai cerné trois approches théoriques:

(a) La sociologie générale: Georg Simmel nous fournit deux éléments importants. Il a prouvé que la « limitation» est nécessaire pour régler la participation aux activités collectives. L'espace donné exigerait une telle limitation des intérêts. La conséquence en est qu'il existe une division entre ceux qui en font partie et les autres qui restent. À partir de son analyse de l'action réciproque entre des groupes migratoires et leurs conditions sociales dans l'espace, Simmel nous conduit à vérifier si les frontaliers forment un groupe migratoire spécifique, avec une structure particulière, et quels effets résultent de leur action transfrontalière sur « le groupe sédentaire». Pierre Bourdieu (1979) envisage aussi les activités et les comportements sociaux sous un aspect spatial. L'espace social positionne l'agent selon ses différents mouvements, stratégies et trajectoires. D'un autre côté, la théorie de la distinction de Bourdieu est plutôt une théorie des différentes façons qu'ont les acteurs de tracer leurs limites et frontières. Mais la connaissance des mécanismes et des logiques des acteurs nous permet de mieux comprendre l'action de franchissement d'une frontière-limite. Quelle stratégie est à la disposition du frontalier pour équilibrer les attentes de son monde vécu et les obligations liées au système de travail d'un pays étranger? Une recherche future sur les frontaliers devrait aussi appliquer systématiquement la théorie des goûts à l'analyse des modes de vie frontaliers.

(b) La phénoménologie ethnographique et la sociologie culturelle de la frontière font souvent de belles descriptions des frontaliers en tant que «cas extraordinaires », par exemple des contrebandiers, des espions, des voleurs traversant une frontière. R. Girtler $(1992$; 1995) analyse ces cas extraordinaires en cherchant à découvrir leurs systèmes de subculture. De la même façon, on peut se demander quels sont les «supports» (Castel, 1995) qui garantissent au frontalier «d'exister comme tel»(Castel, Haroche, 2001, p. 13). Autrement dit, il nous faut une meilleure connaissance des ressources dont dispose l'individu qui se décide ou bien qui est contraint de «faire frontière».

(c) La sociologie des migrations ne reconnaît pas le type du frontalier dans la classification des migrants, remigrants, migrants de dispersion et des transmigrants. Le critère d'exclusion est que le frontalier ne reste pas «durablement» dans l'autre pays. Par contre, la sociologie du travail nous livre des informations utiles pour la construction d'une typologie du frontalier. Il s'agit d'abord d'une catégorie juridico-administrative et spécifique de travailleur. La notion même de frontalier est déjà préconstruite par le décret de l'Union Européenne cité 
ci-dessus. Son statut est déterminé par l'emploi à l'étranger et le domicile fixe dans un autre État. Étant donné leur situation d'emploi, on peut dire que leurs rapports de travail sont précaires. Une revalorisation de leur qualification professionnelle est plutôt rare.

\section{UNE FIGURE HYBRIDE?}

Pour résumer: «faire frontière » est une action sociale spécifique qui se réalise dans deux espaces géographiques séparés par des frontières nationales et socioculturelles. La situation économique et le marché de l'emploi de «chez eux» pousse en général les frontaliers hors de leur territoire habituel. Les espaces traversés ont différentes valences. Il est évident que chez eux, le petit monde connu, remplit la fonction d'une «région revers » (A. Giddens) ou bien, d'après J. Habermas, du monde vécu. Que ce dernier soit instable à cause du travail frontalier n'est pas prouvé. Quelques indices soutiennent au contraire l'hypothèse d'une plus grande stabilité de la structure du monde vécu en Lorraine que dans la Sarre. J'expliquerais ce phénomène par le fait que le système juridique qui régit le statut de frontalier lui confère une situation hybride: travailleur étranger ailleurs et citoyen en France. Il peut se retirer dans ce petit monde qui n'est pas directement exposé aux influences du système de travail. Mais l'action frontalière de cette figure hybride reste très risquée puisque son succès ne se calcule pas en raison d'une situation économique et politique instable.

«Faire frontière » dans le cas du frontalier lorrain est le résultat d'un processus de modernisation des régions frontalières. Etre frontalier représente une forme d'existence moderne.

Universität des Saarlandes, Saarbrücken

hl.kraemer@mx.uni-saarland.de

\section{RÉFÉRENCES BIBLIOGRAPHIQUES}

Andruchowytsch J., Das letzte Territorium, Frankfurt a. M., Suhrkamp-Verlag, 2003.

Besnard Ph., Moeurs et humeurs des Français au fil des saisons, Paris, Balland, 1989.

Bourdieu P., La distinction, Paris, Editions de Minuit, 1979.

Brücher W., H. P. Dörrenbächer, Grenzüberschreitende Beziehungen zwischen dem Saarland und Lothringen-Ausdruck einer Mischkultur?, in: R.Marti (sous la dir. de), Grenzkultur-Mischkultur?, Saarbrücken, SDV-Verlag, 2000.

CDR/ISOPLAN, Grenzgängerinnen im Raum Saarland-Lothringen, Metz und Saarbrücken, ISOPLAN, 1996.

EURES-TRANSFRONTALIER, Regionalprofil Saar-Lor-Lux-Rheinland-Pfalz, Saarbrücken, EURES, 2001.

Castel R., Les métamorphoses de la question sociale, Paris, Fayard, 1995.

Castel R., C. Haroche, Propriété privée, propriété sociale, propriété de soi, Paris, Fayard, 2001.

Girtler R., Schmuggler: Von Grenzen und ihren Überwindern, Linz, Veritas, 1992.

Girtler, R., Randkulturen: Theorien der Unanständigkeit, Wien-Köln, Böhlau, 1995.

Glucksmann A., Le Bien et le Mal, Paris, Laffont, 1997 
Gries M. et alii, Frankreichbezüge und grenzüberschreitender Qualifizierungsbedarf, Saarbrücken, IFB, 1997.

Gulden A., Nur auf der Grenze bin ich zu Haus, in: R. Faber, B. Naumann (sous la dir. de), Literatur der Grenze, Würzburg, Königshausen \& Neumann, 1995.

Krämer H. L., Grenzgänger aus soziologischer Sicht, in: R. Schneider (sous la dir. de), Grenzgänger, Saarbrücken, SDV-Verlag, 1998.

Lefebvre L., Der Rhein und seine Geschichte, Frankfurt a. M., Suhrkamp-Verlag, 1994.

Mendras H., L'Europe des Européens, Paris, Gallimard, 1997.

Pankoke E., Soziokulturelle Grenzgänger, in: H.Schwengel (sous la dir. de), Grenzenlose Gesellschaft?, Pfaffenweiler, Centaurus-Verlag, 1999, Bd II/1 Sektionen, pp. 150-154.

Roth J., Briefe aus Deutschland, Blieskastel, Gollenstein-Verlag, s.d.

Schütz A., Der Fremde: ein sozialpsychologischer Versuch, in: A.Schütz, Gesammelte Aufsätze, Den Haag, M. Nijhoff, 1972, Bd 2.

Simmel G., Soziologie, Leipzig, Duncker \& Humblot, 1908.

Sztompa P., Vertrauen, Kölner Zeitschrift für Soziologie und Sozialpsychologie, 35, 1995, pp. 254-276. 